\title{
Why Hypothetical Syllogism is Invalid for Indicative Conditionals
}

Moti Mizrahi

St. John's University

[Forthcoming in Thought: A Journal of Philosophy]

Abstract: In this paper, I present a schema for generating counterexamples to the argument form known as Hypothetical Syllogism (HS) with indicative conditionals. If my schema for generating counterexamples to HS works as I think it does, then HS is invalid for indicative conditionals.

Keywords: counterexample; formal validity; hypothetical syllogism; indicative conditional

\section{Introduction}

In standard propositional logic, the argument form known as Hypothetical Syllogism (HS) is often taken to be formally valid (see, e.g., Copi, Cohen, and Flage 2007, p. 227):

If $P$, then $Q$.
If $Q$, then $R$.

Therefore, if $\mathrm{P}$, then $\mathrm{R}$. 
To say that an argument form is valid is to say that the premises, if true, necessitate the truth of the conclusion. If $\mathrm{HS}$ is formally valid, then no matter what we substitute for $\mathrm{P}$, $\mathrm{Q}$, and $\mathrm{R}$, an argument that has the form HS will be valid.

The problem is that there are putative counterexamples to HS. That is to say, there are arguments of the HS form that seem to have true premises and a false conclusion. For example, one putative counterexample to HS can be found in Wright (1983, pp. 134140):

(W1) If there had been snow in the valley yesterday, I would have gone skiing.

(W2) If an avalanche had then been taking place, there would have been snow in the valley yesterday.

(W3) Therefore, if an avalanche had been taking place yesterday, I would have gone skiing.

In this case, it seems that (W3) can be false even if (W1) and (W2) are true. Wright himself does not seem to think that this is a genuine counterexample to HS. He seems to think that the invalidity of HS is apparent, not real, because of subtle changes in context. (Cf. Brogaard and Salerno 2008.)

Another putative counterexample to HS can be found in Stalnaker (1968, pp. 98-112):

(S1) If Hoover had been born in Russia, he would have been a communist. 
(S2) If Hoover had been a communist, he would have been a traitor.

(S3) Therefore, if Hoover had been born in Russia he would have been a traitor.

In this case, (S3) seems false, even though (S1) and (S2) seem true. This putative counterexamples, however, is also somewhat controversial. Lowe (1990, pp. 80-87), for instance, argues that it is more plausible that Stalnaker's argument commits the fallacy of equivocation than that HS is invalid.

More recently, Morreau (2009, pp. 447-464) has offered the following putative counterexample to HS:

(M1) If there was a thunderstorm, it rained.

(M2) If it rained, there was an ordinary rain shower, not a thunderstorm.

(M3) Therefore, if there was a thunderstorm, there was an ordinary rain shower, not a thunderstorm.

In light of such putative counterexamples, our options, according to Morreau (2009, p. 449), are as follows: "either we say that the hypothetical syllogism is valid, and explain away the paradoxes; or else we say that it is not valid, and explain why counterexamples are scarce."

In what follows, I argue that counterexamples to HS are not scarce. In fact, they can be generated quite easily. For the purposes of this paper, I will focus on HS with indicative (or "straight") conditionals ('If $P$, then $Q$ ') regimented as 'P $\rightarrow Q$ ', as opposed to 
subjunctive conditionals ('If it were that $P$, then it would be that $Q$ '), which Bennett regiments using the horseshoe rather than the arrow (see Bennett 2003, pp. 13-15). I will assume the following about indicative conditionals:

(MP) ' $P \rightarrow Q$ ' is false whenever $P$ is true and $Q$ is false.

In other words, I will assume that Modus Ponens is truth-preserving for indicative conditionals of the form 'P $\rightarrow$ Q'. (Cf. Lewis 1973, p. 132.) My counterexamples, then, are intended to be counterexamples to the following argument form:

$$
\begin{aligned}
& P \rightarrow Q \\
& Q \rightarrow R \\
& \therefore P \rightarrow R
\end{aligned}
$$

\section{A schema for generating counterexamples to HS}

Here is a counterexample to HS:

$\mathrm{P}=\mathrm{I}$ am in Boston at time $t$.

$\mathrm{Q}=\mathrm{I}$ am in a city whose name starts with the letter $\mathrm{B}$ at time $t$.

$\mathrm{R}=\mathrm{I}$ might be in Baltimore at time $t$.

(T1) If I am in Boston at time $t$, then I am in a city whose name starts with the letter B at time $t$.

(T2) If I am in a city whose name starts with the letter B at time $t$, then I might be in Baltimore at time $t$. 
(T3) Therefore, if I am in Boston at time $t$, then I might be in Baltimore at time $t$. In this case, I submit, (T1) and (T2) are true, whereas (T3) is false. If I am in Boston at time $t$, then it is not the case that I might be in Baltimore at the same time, since I am already in Boston at that time, and thus could not be in Baltimore at the same time. Once one is already in spatial location $L_{1}$ at time $t$, the window of physical possibilities is closed, as it were, for being in another spatial location $L_{2}$ at time $t$, since a human person cannot be in two different spatial locations simultaneously. If this is correct, then the antecedent of (T3) is true but the consequent is false, which means that (T3) is false by assumption (MP).

My argument for the claim that (T3) is false, then, is the following:

1. If being in two different spatial locations at the same time is physically impossible for a human person $S$, then, if $S$ is already in $L_{1}$ at time $t$, it is not the case that $S$ might be in $L_{2}$ at time $t$ as well.

2. Being in Boston and being in Baltimore at the same time is physically impossible for a human person.

3. Therefore, if $S$ is already in Boston at time $t$, it is not the case that $S$ might be in Baltimore at time $t$ as well.

If this argument is sound, then the argument with premises (T1) and (T2) and conclusion (T3) constitutes a counterexample to HS.

Additional counterexamples to HS can be generated using the same schema. For example: 
$\mathrm{P}=\mathrm{I}$ am watching Pulp Fiction at time $t$.

$\mathrm{Q}=\mathrm{I}$ am watching a movie whose name starts with the letter $\mathrm{P}$ at time $t$.

$\mathrm{R}=\mathrm{I}$ might be watching Planet of the Apes at time $t$.

(F1) If I am watching Pulp Fiction at time $t$, then I am watching a movie whose name starts with the letter $\mathrm{P}$ at time $t$.

(F2) If I am watching a movie whose name starts with the letter $\mathrm{P}$ at time $t$, then I might be watching Planet of the Apes at time $t$.

(F3) Therefore, if I am watching Pulp Fiction at time $t$, then I might be watching Planet of the Apes at time $t$.

In this case, I submit, (F1) and (F2) are true, whereas (F3) is false. If I am watching Pulp Fiction at time $t$, then it is not the case that I might be watching Planet of the Apes at the same time, since I am already watching Pulp Fiction at that time, and thus could not be watching Planet of the Apes at the same time. Once one is already watching one film at time $t$, the window of physical possibilities is closed, as it were, for watching another film at time $t$, since a human person cannot watch two different movies simultaneously. In other words, one can direct one's gaze at one screen or another, but not both simultaneously. So, even if Pulp Fiction is playing on my laptop, and Planet of the Apes is playing on my iPad at the same time, I would still have to shift my gaze from one device to the other in order to watch the movie that is playing on that device at a particular time. If this is correct, then the antecedent of $(F 3)$ is true but the consequent is false, which means that (F3) is false by assumption (MP).

My argument for the claim that (F3) is false, then, is the following: 
1. If watching two different movies at the same time is physically impossible for a human person $S$, then, if $S$ is already watching $M_{1}$ at time $t$, it is not the case that $S$ might be watching $M_{2}$ at time $t$ as well.

2. Watching Pulp Fiction and Planet of the Apes at the same time is physically impossible for a human person.

3. Therefore, if $S$ is already watching Pulp Fiction at time $t$, it is not the case that $S$ might be watching Planet of the Apes at time $t$ as well.

If this argument is sound, then the argument with premises (F1) and (F2) and conclusion (F3) constitutes a counterexample to HS.

Using this schema, I submit, additional counterexamples to HS can be generated. If this is correct, then, contrary to what Morreau (2009, p. 449) says, one can argue that HS is invalid without having to explain why counterexamples are scarce, since they are not scarce.

\section{Conclusion}

In this paper, I have presented a schema for generating counterexamples to HS with indicative conditionals. If this schema for generating counterexamples to HS with indicative conditionals works as I think it does, then one can argue that HS is invalid without having to explain why counterexamples are scarce, since they are not scarce. 


\section{Acknowledgments}

I am grateful to an anonymous reviewer of Thought for very helpful comments on an earlier draft.

\section{References}

Bennett, J. (2003). A Philosophical Guide to Conditionals. New York: Oxford University Press.

Brogaard, B. and Salerno, J. (2008). Counterfactuals and Context. Analysis, 68, 39-46.

Copi, I. M., Cohen, C., and Flage, D. E. (2007). Essentials of Logic. Second Edition. Upper Saddle River, NJ: Pearson Prentice Hall.

Lewis, D. K. (1973). Counterfactuals. Cambridge, MA: Harvard University Press.

Lowe, E. J. (1990). Conditionals, Context and Sensitivity. Analysis, 50, 80-87.

Maclachlan, D. L. C. (1970). The Pure Hypothetical Syllogism and Entailment. The Philosophical Quarterly, 20, 26-40.

Morreau, M. (2009). The Hypothetical Syllogism. Journal of Philosophical Logic, 38, 447-464.

Stalnaker, R. (1968) A Theory of Conditionals. In Studies in Logical Theory: American Philosophical Quarterly Monograph Series, no. 2, ed. Nicholas Rescher, pp. 98-112. Oxford: Blackwell.

Wright, C. (1983). Keeping track of Nozick. Analysis, 43, 134-140.

Wright, C. (1984). Comment on Lowe. Analysis, 44, 183-185. 